\title{
INFECÇÕES SEXUALMENTE TRANSMISSÍVEIS EM HOMENS PRIVADOS DE LIBERDADE: UM RELATO DE EXPERIÊNCIA
}

\section{Jorge Luís Silveira Marques'; Márcia Gabriela Rodrigues de Lima²; Giulliane Ramos Londero'; Guilherme Albarello Weber ${ }^{4}$}

\section{RESUMO}

Objetivamos, com esse trabalho, analisar a incidência de ISTs em homens privados de liberdade, em uma penitenciária da cidade de Santa Maria - RS, que realizaram testagem rápida. Para tanto, apresentamos uma análise acerca dos relatórios e descrevemos as ações desenvolvidas com a inserção do Programa de Residência Multiprofissional em Atenção Clínica Especializada com ênfase em Infectologia e Neurologia (UFN) dentro do sistema carcerário, bem como os desafios que se configuraram ao trabalho e ao processo de formação profissional a partir desse contexto. De acordo com os dados, foram realizadas 8 visitas no primeiro semestre deste ano, sendo testados 71 apenados. Conclui-se que é necessário haver o fortalecimento nas ações de promoção, prevenção e educação em saúde dentro do sistema carcerário, sendo a informação utilizada como instrumento de trabalho.

Palavras-chave: Educação em Saúde; População privada de liberdade; Política de Atenção à Saúde; Doenças sexualmente transmissíveis.

Eixo Temático: Atenção Integral e Promoção à Saúde (AIPS)

\section{INTRODUÇÃO}

Estima-se que ocorram cerca de 500 milhões novos casos de infecções sexualmente transmissíveis curáveis no mundo a cada ano, afetando homens e mulheres entre 15 e 49 anos, dos quais cerca de 100.000 a 12 milhões ocorrem no Brasil. No primeiro semestre de 2017, o número de pessoas privadas de liberdade no Brasil foi de 726.354. Em relação aos anos anteriores, a taxa de crescimento

\footnotetext{
1 Jorge Luís Silveira Marques - Universidade Franciscana (UFN) - jorge.marques@ufn.edu.br.

2 Márcia Gabriela Rodrigues de Lima - Secretaria Municipal da Saúde (SMS) grlmarcia@yahoo.com.br.

${ }^{3}$ Giulliane Ramos Londero - Universidade Franciscana (UFN) - g.londero@ufn.edu.br.

${ }^{4}$ Guilherme Albarello Weber - Universidade Franciscana (UFN) - quilherme.weber@ufn.edu.br
} 
dessa população tem diminuído (OMS, 2012; BRASIL, 2019).

As infecções sexualmente transmissíveis são um dos problemas de saúde mais comuns no mundo e sua gravidade ainda é desconhecida, pois, nesse grupo de infecções, poucas são relatadas sistematicamente e, quando os dados são menos organizados, envolvem apenas sífilis e AIDS. Embora não estejam disponíveis informações mais precisas sobre a verdadeira extensão das infecções sexualmente transmissíveis, sua incidência e prevalência são consideradas altas e as consequências excedem a intimidade da vida sexual privada, representando um sério problema de saúde em todo o mundo, isto é, uma importante causa de mortalidade (BRASIL, 2020).

Nas últimas décadas, muitos estudos mostraram que o padrão das infecções sexualmente transmissíveis (ISTs) varia entre as diferentes populações. Dentre elas, a população carcerária se destaca em escala global porque a prevalência dessas infecções é bastante elevada. Em razão disso, configura-se como um grupo de risco potencial (STIEF et al., 2010; SILVA et al., 2017; SOARES et al., 2019).

A Política Nacional de Atenção Integral à Saúde da Pessoa Privada de Liberdade (PNAISP) prevê:

[...] ações de promoção à saúde e prevenção de agravos no sistema prisional, das pessoas privadas de liberdade ao longo do percurso penitenciário e dos profissionais que atendem infratores, familiares e outros relacionados ao sistema, por exemplo, voluntários. Para o alcance desta política, entendemos por sistema prisional todo o itinerário carcerário, desde - momento da detenção do cidadão e sua condução para um estabelecimento policial até a finalização do cumprimento da pena. Entendem-se ainda por pessoa privada de liberdade no sistema prisional os indivíduos maiores de 18 anos custodiados em unidades prisionais (excluem - se os tutelados pelo Sistema Nacional Socioeducativo - Sinase). Na população do sistema prisional é possível encontrar grande pluralidade: homens jovens, em sua maioria; estrangeiros; idosos; mulheres; crianças (filhos dessas mulheres privadas de liberdade) e populações vulneráveis, como indígenas, Lésbicas Gays, Bissexuais e Transexuais (LGBT), pessoas com transtornos mentais e com deficiências. Todos os tipos de agravos em saúde que acometem a população geral também são encontrados no sistema prisional, mas podem ser potencializados devido às condições precárias de confinamento de grande parte das unidades prisionais e também à superlotação. Portanto, nesse cenário, é obrigatório que as políticas públicas sejam transversais, de forma a atender a todos em todas as suas especificidades (BRASIL, 2014, p. 07). 
O Programa de Residência Multiprofissional constitui-se como uma PósGraduação Lato sensu de inserção nas políticas públicas de saúde com a integração do Ministério da Saúde e Ministério da Educação, sendo criada por meio da promulgação da Lei n 11.129 de 2005. A ênfase em Atenção Clínica Especializada com ênfase em Infectologia e Neurologia é uma das áreas abrangidas pelo Programa de Residência Multiprofissional da Universidade Franciscana de Santa Maria - RS.

Diante desse contexto, a presente produção apresenta um relato de experiência de um fisioterapeuta do primeiro ano de Residência Multiprofissional em Atenção Clínica Especializada com ênfase em Infectologia e Neurologia - UFN, no campo de estágio da Política HIV/AIDS, ISTs e Hepatites Virais, da Secretaria da Saúde de Santa Maria, como parte das atividades inerentes ao campo de atuação dos profissionais da saúde inseridos na residência.

Objetiva-se, com este estudo, analisar a incidência de ISTs em homens privados de liberdade na Penitenciária Estadual de Santa Maria (PESM), a partir dos resultados das ações de testagem rápida para infecções sexualmente transmissíveis.

\section{METODOLOGIA}

Trata-se de uma produção descritiva, do tipo relato de experiência, elaborado no contexto de práticas de ensino-aprendizagem, através do Programa de Residência Multiprofissional em Atenção Clínica Especializada com ênfase em Infectologia e Neurologia da Universidade Franciscana de Santa Maria, a partir de ações desenvolvidas no campo de estágio na Política HIV/AIDS, ISTs e Hepatites Virais da Secretaria de Saúde. Nesse empreendimento, foi realizada uma análise documental, no livro de registro e controle de resultados dos testes rápidos, referente ao primeiro semestre de 2021, no qual totalizaram 71 apenados atendidos.

Referente ao tipo, são ofertadas testagens para rastreamento do HIV, SÍFILIS, HBsAg e HCV, nas terças-feiras, pela manhã, em uma sala do Ambulatório de Saúde, dentro da Penitenciária. Os profissionais que realizam esse procedimento são vinculados à Política HIV/AIDS, ISTs e Hepatites Virais e/ou da SAE/CTA Casa Treze de Maio. 
$\mathrm{Na}$ PESM, os apenados são todos do sexo masculino e cumprem pena de reclusão em regime fechado, por isso, a casa prisional fica localizada a $15 \mathrm{~km}$ do centro da cidade, com o intuito de dificultar o seu acesso, sendo que há uma demanda de agendamento do transporte específico para o deslocamento da equipe até o local.

Em geral, os detentos são questionados sobre a existência de conhecimento prévio dos testes ou mesmo se já realizaram a testagem em algum momento. Enquanto são esperados os resultados dos testes, é realizada uma orientação em relação ao período de janela imunológica para as ISTs, às formas de contágio do HIV, sífilis e hepatites, às formas de prevenção com uso de preservativo interno e externo, ao não compartilhamento de objetos perfuro cortante para minimizar a transmissão e o contágio, principalmente, por Hepatite C; entre outras orientações.

\section{RESULTADOS E DISCUSSÕES}

De acordo com as variáveis em estudo, os resultados foram apresentados em duas categorias. A primeira parte diz respeito às variáveis independentes: idade e etnia. Na segunda categoria, são apresentados dados sobre o número de testagens e os casos reagentes.

Na PESM, são abrigados cerca de 1.100 apenados, sendo que destes, 71 foram testados no primeiro semestre de 2021, durante oito visitas ao estabelecimento prisional. Sobre o perfil dos privados de liberdade testados, sua faixa etária está entre 18 e 60 anos ou mais, com média de 34,8 anos de idade, com maior incidência em apenados de 18 a 44 anos, somando um número de 58, conforme a (Tabela 1). Além disso, na Tabela 1 também são apresentadas questões relacionadas à raça da amostra, resultando em 41 apenados $(57,7 \%)$ autodeclarados brancos, $21(29,6 \%)$ pardos, $8(11,3 \%)$ pretos, $1(1,4 \%)$ indígenas. 


\section{QUFN}

Tabela 1 - Características sociodemográficas da amostra

\begin{tabular}{llc}
\hline Variáveis & \multicolumn{2}{c}{ Características da amostra } \\
& N & $\%$ \\
\hline Idade & 29 & $40,8 \%$ \\
$18-30$ & 29 & $40,8 \%$ \\
$31-44$ & 11 & $15,5 \%$ \\
$45-59$ & 2 & $2,9 \%$ \\
$60+$ & & \\
Raça & 0 & $0 \%$ \\
Amarelo & 41 & $57,7 \%$ \\
Branco & 1 & $1,4 \%$ \\
Indígena & 21 & $29,6 \%$ \\
Pardo & 8 & $11,3 \%$ \\
Preto & & \\
\hline Total & 71 & $100 \%$ \\
\hline
\end{tabular}

Fonte: Política HIV/AIDS, ISTs e Hepatites Virais de Santa Maria

Nota: Relatório Semestral 2021 de Ações desenvolvidas pela política

Os dados acima corroboram com os números nacionais (BRASIL, 2017), pois em 2016, o maior número de pessoas privadas de liberdade (notificadas) se autodeclarava branco, seguidas de pessoas autodeclaradas pardas e pessoas autodeclaradas pretas, desmistificando a predominância do quesito raça/cor preto no sistema prisional. Relacionado a faixa etária, o resultado reforçou o estudo de Negreiros e Vieira (2017), no qual é apontado que a maioria da população carcerária masculina brasileira está entre jovens e adultos, salientando que a população vem apresentando um novo padrão sociodemográfico, caracterizado pelo aumento populacional e por transformações na composição de sua estrutura etária, com um significativo aumento no número de jovens (CABRAL, 2014).

No que concerne à Tabela 2, foram realizados 281 testes rápidos para ISTs, resultando em 12 casos reagentes, sendo eles 7 para sífilis, 3 para hepatite $C, 2$ para HIV (foram realizados 3 testes a menos para HIV, pois os apenados testados já sabiam seu diagnóstico para o HIV, não demandando nova testagem). 
Tabela 2 - Incidência de casos reagentes na população testada

\begin{tabular}{lcc}
\hline Variáveis & \multicolumn{2}{c}{ Características da amostra } \\
& No de testagens & Casos reagentes \\
\hline HIV & 68 & 2 \\
Sífilis & 71 & 7 \\
HbAsg & 71 & 0 \\
HCV & 71 & 3 \\
& & \\
\hline Total & 281 & 12
\end{tabular}

Fonte: Política HIV/AIDS, ISTs e Hepatites Virais de Santa Maria

Nota: Relatório Semestral 2021 de Ações desenvolvidas pela política

A Tabela 2 demonstra uma tendência maior para o número de casos de Sífilis, quando comparado ao de HIV e Hepatites Virais B/C. Essa infecção, por ser sistêmica e de evolução crônica, embora o tratamento seja fácil e eficaz, ainda desafia a saúde pública no seu controle e erradicação (AGUIAR et al., 2021).

Em relação ao HIV, Reis e Bernardes (2011) apontam que o quantitativo de infectados nos sistemas prisionais varia de 3 a $6 \%$. Entretanto, os dados disponíveis podem estar subnotificados, pois muitos presos possuem HIV reagente, mas não foram diagnosticados. Além disso, muitos sistemas prisionais não dispõem de serviços de saúde e as dificuldades de deslocamento do detento para além dos presídios tornam ainda mais difícil o acesso dos presos aos serviços de saúde.

A incidência de IST/HIV em detentos é duas vezes maior do que na população que vivem em sociedade, livremente. Isso ocorre devido ao confinamento que favorece as relações afetivas sem o uso de preservativo, além da violência sexual praticada por parte de outros detentos, bem como o compartilhamento de lâminas de barbear e agulhas, propícias para a transmissão e contágio de hepatite $B$ e C (FERNANDES et al., 2016).

Observou-se, nessa direção, durante os atendimentos, que há pouco conhecimento em relação às ISTs quanto a sua transmissão, tratamento e sintomas. Como comportamentos de risco, destacam-se: a não utilização de preservativo, a quantidade de parceiros sexuais, o uso de álcool e outras drogas durante relação sexual, entre outros (SALES, 2016). Em uma das penitenciárias localizadas em São Paulo (Zona Oeste), no ano de 2017, uma pesquisa que considerou 1.141 presos do 
sexo masculino, dos quais, mesmo que em minoria, os participantes tiveram dúvidas em relação à forma de contágio da gonorreia, sífilis e HPV. Isso está relacionado, sobretudo, ao desconhecimento da forma de contágio de uma doença, o que possui implicações relevantes em relação à sua prevenção (CARVALHO et al., 2020).

A prevenção representa uma estratégia básica para o controle da transmissão das ISTs e, para que seja assegurada, é imprescindível a realização de atividades educativas que priorizem a percepção de risco, o estímulo a mudança no comportamento sexual, a promoção e adoção de medidas preventivas com ênfase na utilização adequada do preservativo (FERNANDES et al.; 2016).

Dada a vulnerabilidade dessa população, métodos diferenciados voltados para nortear a detecção e o tratamento de doenças são considerados de extrema importância. Isso pode ser alcançado por meio de ações de educação em saúde, consultas e diversas outras formas de divulgação de informações. Conforme Serra e Lima (2019), a educação em saúde é uma forma de capacitação do indivíduo no que concerne ao autocuidado, o que garante e promove sua saúde.

\section{CONCLUSÃO}

O presente estudo demonstrou que a população de privados de liberdade possui múltiplos fatores de risco que predispõem à transmissão de ISTs, sendo os principais deles relacionados a não adesão ao preservativo nas relações sexuais e à falta de informação frente às infecções sexualmente transmissíveis. Diante disso, é evidenciada a importância da atuação multiprofissional em saúde, sobretudo, por meio do diagnóstico precoce por testagem rápida, das orientações e demais ações que visem à prevenção de doenças à promoção e manutenção da saúde, respeitando a singularidade das pessoas privadas de liberdade, dentro do sistema prisional.

Conclui-se, então, que é necessário o fortalecimento das ações de promoção e educação em saúde dentro do sistema carcerário, com realização de campanhas por meio de rodas de conversa, educação sexual da população e disponibilização de cartilhas que explicitem as infecções sexualmente transmissíveis e, principalmente, a importância da prevenção, que se dá por meio do preservativo e outros meios de prevenção, assim como, conjuntamente, a implementação da Política Nacional de 
Atenção Integral à Saúde das Pessoas Privadas de Liberdade no Sistema Prisional (PNAISP) no município de Santa Maria.

\section{REFERÊNCIAS}

AGUIAR, B. M. et al. Vulnerabilidade às infecções sexualmente transmissíveis de adolescentes privados de liberdade. Brazilian Journal of Health Review, Curitiba, v. 4, n. 1, p. 2666-2675, 2021. Disponível em: <https://www.brazilianjournals.com/index.php/BJHR/article/view/24473>.

BRASIL. Ministério da Saúde. Política Nacional de Atenção Integral à Saúde das Pessoas Privadas de Liberdade no Sistema Prisional/ Ministério da Saúde, Secretaria de Atenção à Saúde, Departamento de Ações Programáticas Estratégicas, Coordenação de Saúde no Sistema Prisional. Brasília, 2014. Disponível em:

$<$ http://www.as.saude.ms.gov.br/wpcontent/uploads/2016/06/Cartilha-PNAISP.pdf>.

BRASIL. Ministério da Saúde. Boletim Epidemiológico/Secretaria de Vigilância em Saúde, v. 48, n. 36, 2017. Disponível em: $<$ https://portaldeboaspraticas. iff.fiocruz.br/biblioteca/boletim-epidemiologico-volume48-n-36-2017/>.

BRASIL. Ministério da Justiça e Segurança Pública. Levantamento Nacional de informações penitenciárias, atualização junho. Departamento Penitenciário Nacional, 2019.

Disponível

em:

$<$ http://antigo.depen.gov.br/DEPEN/depen/sisdepen/infopen/relatorios-

sinteticos/infopen-jun-2017-rev-12072019-0721.pdf>.

BRASIL. Ministério da Saúde. Protocolo Clínico e Diretrizes Terapêuticas para Atenção Integral às Pessoas com Infecções Sexualmente Transmissíveis (IST). Brasília, 2020. Disponível em: <http://www.aids.gov.br/pt-br/pub/2015/protocoloclinico-e-diretrizes-terapeuticas-para-atencao-integral-pessoas-com-infeccoes>.

CABRAL, R. P. Reintegração social em Goiás: o perfil do apenado e a atuação do patronato em prol do egresso. 2014. 109 p. Dissertação (Mestrado em Direitos Humanos) - Universidade Federal de Goiás, Goiânia, GO, 2014. Disponível em: $<$ https://files.cercomp.ufg.br/weby/up/788/o/Ruth-do-Prado-Cabral.pdf>. 
CARVALHO, F. F. et al. Conhecimento da população privada de liberdade sobre infecções sexualmente transmissíveis. Revista Gaúcha de Enfermagem, v. 41, p. $1-9$, 2020.

Disponível

em:

$<$ https://www.scielo.br/j/rgenf/a/sdJn8Vng4tRBmqJWrYxm3CL/?lang=pt>.

FERNANDES, M. A. et al. Infecções sexualmente transmissíveis e as vivências de mulheres em situação de reclusão. Rev Enferm, v. 24, n. 6, p. 1-6, 2016. Disponível em:

$<$ https://www.e-

publicacoes.uerj.br/index.php/enfermagemuerj/article/view/27774>.

NEGREIROS, D. E. H.; VIEIRA, D. S. Prevalência de hepatites b, c, sífilis e hiv em privados de liberdade - Porto Velho, Rondônia. Rev Interd, v. 10, n. 1, p. 43-52, 2017. Disponível em: < https://dialnet.unirioja.es/servlet/articulo?codigo=6771887 > .

OMS, World Bank Special Programme of Research, Development and Research Training in Human Reproduction \& World Health Organization. (2012). Sexually transmitted infections (STIs): the importance of a renewed commitment to STI prevention and control in achieving global sexual and reproductive health. World Health Organization, p. 1-8. 2012. Disponível em: $<$ https://apps.who.int/iris/bitstream/handle/10665/75838/WHO RHR 12.31 eng.pdf? sequence $=1$ \&isAllowed $=y>$.

REIS, C. B.; BERNARDES, E. B. O que acontece atrás das grades: estratégias de prevenção desenvolvidas nas delegacias civis contra HIV/AIDS e outras doenças sexualmente transmissíveis. Ciência \& Saúde Coletiva, v. 16, n. 7, p. 3331-3338, 2011. Disponível em: <https://www.scielo.br/pdf/csc/v16n7/32.pdf>.

SALES, W. B. et al . Comportamento sexual de risco e conhecimento sobre IST/SIDA em universitários da saúde. Rev. Enf. Ref., Coimbra , v. 4, n. 10, p. 1927 , 2016.

Disponível

em

$<$ http://www.scielo.mec.pt/scielo.php?script=sci arttext\&pid=>.

SILVA, A. A. S. et. al. Prevalência de hepatite B e fatores associados em internos de sistema prisional. Acta Paulista de Enfermagem, São Paulo, v. 30, n. 1, pág. 6672 , 2017. Disponível

em:

$<$ https://www.scielo.br/j/ape/a/CtHQtKx3hFsGXLdVVq96rVn/?lang=pt\&format=pdf $>$. 
SERRA, A. G.; LIMA, R. R. Promoção da saúde para pessoas no regime semiaberto do sistema penitenciário: relato de experiência. Rev. Saúde debate, Rio de Janeiro, v. $43, \quad$ n. $123, \quad$ p. 1270-1281, 2019. Disponível em: $<$ https://www.scielosp.org/pdf/sdeb/2019.v43n123/1270-1281/pt>.

SOARES et al. Sífilis em privados de liberdade em uma unidade prisional no interior de Rondônia. Brazilian Journal of Health Review, Curitiba, v. 2, n. 2, pág. 21952205 , 2019.

Disponível

em:

$<$ https://www.brazilianjournals.com/index.php/BJHR/article/view/1632/1506>.

STIEF, A. C. F et. al. Seroprevalence of hepatitis B virus infection and associated factors among prison inmates in state of Mato Grosso do Sul, Brazil. Revista da Sociedade Brasileira de Medicina Tropical, Uberaba, v. 43, n. 5, p. 512-515, 2010. Disponível em:

http:

$<$ https://www.scielo.br/j/rsbmt/a/jsm4QdsMdsmfRxfwqTJDYNc/?lang=en>. 